\title{
Natural killer cells control a T-helper 1 response in patients with Behçet's disease
}

\author{
Yukie Yamaguchi1,2, Hayato Takahashi³, Takashi Satoh1', Yuka Okazaki1, Nobuhisa Mizuki, Kazuo Takahashi², \\ Zenro Ikezawa² and Masataka Kuwana*1
}

\begin{abstract}
Introduction: Behçet's disease (BD) is a multisystem inflammatory disorder, in which a T-helper 1 (Th1)-polarized immune response plays a major role in the pathogenic process. We evaluated the regulatory role of natural killer (NK) cells in Th1-biased immune responses in patients with BD.

Methods: We studied 47 patients with $B D$, including 10 with active disease (aBD) and 37 with inactive disease (iBD), and 29 healthy controls. The activation status and cytotoxic activity of NK cells were examined by flow cytometry. The levels of mRNAs for immune modulatory and cytotoxic molecules in NK cells were determined by quantitative PCR. The IL-12 signal strength in NK cells was determined by assessing the phosphorylation state of its downstream component, signal transducer and activator of transduction 4, by immunoblotting. Finally, NK cells' ability to modulate the Th1 response was evaluated by co-culturing NK cells and T cells without cell contact.

Results: $\mathrm{CD} 69^{+}$-activated NK cells were significantly increased in aBD compared with $\mathrm{BD}$ or control samples, although their cytotoxic activities were similar. The iBD NK cells showed downregulated IL-12 receptor $\beta_{2}$ mRNA levels compared with aBD or control NK cells. The increased IL-13 expression was detected in a subset of BD patients: most of them had iBD. The IL-13 expression level in iBD patients was significantly higher than the level in controls, but was not statistically different compared with the level in aBD patients. The gene expression profile in $\mathrm{BD}$ patients was consistent with the NK type 2 phenotype, and the shift to NK type 2 was associated with disease remission. NK cells from iBD patients showed impaired IL-12-induced signal transducer and activator of transduction 4 phosphorylation. Finally, iBD, but not control, NK cells suppressed IFNy expression by aBD-derived CD4+ ${ }^{+}$cells in vitro.

Conclusions: NK cells may control disease flare/remission in BD patients via NK type 2-mediated modulation of the Th1 response.
\end{abstract}

\section{Introduction}

Behçet's disease (BD) is a multisystem inflammatory disorder characterized by recurrent attacks of uveitis, genital ulcers, oral aphtoid lesions, and skin lesions such as erythema nodosum [1]. The etiology of BD remains unclear, but previous studies on the circulating $\mathrm{CD} 4+\mathrm{T}$ cells and affected lesions of BD patients with active disease showed elevated levels of T-helper 1 (Th1) cytokines, such as IFN $\gamma$ and IL-12, indicating that a Th1polarized immune response plays a major role in the pathogenic process [2-4]. In addition, we recently

* Correspondence: kuwanam@sc.itc.keio.ac.jp

1 Division of Rheumatology, Department of Internal Medicine, Keio University School of Medicine, 35 Shinanomachi, Shinjuku-ku, Tokyo 160-8582, Japan Full list of author information is available at the end of the article reported that cytotoxic lymphocytes, including $\mathrm{CD} 8{ }^{+}$and $\gamma \delta$ T cells, are also involved in the pathogenesis of BD via their cytotoxic activity $[5,6]$. Natural killer (NK) cells are another lymphocyte subset with cytotoxic activity, but their reported numbers and cytotoxic activity in both circulation and BD-associated lesions have been inconsistent [7-9].

NK cells have long been regarded as an essential component of innate immunity, based on their nonspecific cytotoxic activity against virus-infected and tumor cells [10]. Recent evidence, however, indicates that NK cells also regulate innate and acquired immune responses through their secretion of soluble factors and/or cell-cell contact [11]. Recently, a classification of NK cells into two functional subsets based on their expression profiles of cytok- 
ines and cytokine receptors has gained wide acceptance [12]: NK type 1 (NK1) cells mainly produce IFNY and IL10 , and express high levels of IL-12 receptor $\beta_{2}$ (IL$12 \mathrm{R} \beta_{2}$ ); while NK type 2 (NK2) cells produce IL-5 and/or IL-13, and express low levels of IL-12R $\beta_{2}$. This NK1/NK2 paradigm has been shown to control pathogenic Th1biased or Th2-biased immune response in several human immune-mediated diseases, such as multiple sclerosis [13], asthma [14], and pemphigus vulgaris [15].

In the present study, we investigated the potential regulatory functions of NK cells in the Th1-biased environment of $\mathrm{BD}$ by evaluating their activation status, gene expression profiles, and functional properties in association with the disease status.

\section{Materials and methods Patients and controls}

We studied 47 patients with BD (19 men and 28 women, aged $47.3 \pm 17.6$ years) who fulfilled the criteria proposed by an International Study Group [16]. Twenty-nine healthy individuals (14 men and 15 women, aged $38.2 \pm$ 12.3 years) provided control samples.

The BD of the patients was classified as active (aBD) in 10 cases and inactive (iBD) in 37 cases at the time of blood sampling. Active disease was defined as flare of characteristic BD symptoms, including severe skin, mucosal, and/or ocular involvement that required introduction or increase of systemic corticosteroids $(\geq 0.5 \mathrm{mg} / \mathrm{kg})$, cyclosporine, and/or infliximab [6]. Five patients who had $\mathrm{aBD}$ at their first examination were re-evaluated after their BD-related symptoms resolved.

All samples were obtained after the patients and control subjects gave their written informed consent, approved by the International Review Boards of Keio University and Yokohama City University.

\section{HLA-B51 typing}

The presence or absence of HLA-B51 was determined by PCR of the genomic DNA using sequence-specific primers and sequence-based typing [17].

\section{Cell preparations}

Peripheral blood mononuclear cells (PBMCs) were isolated from heparinized venous blood by Lymphoprep (Fresenius Kabi Norge AS, Oslo, Norway) density-gradient centrifugation. NK cells were purified by the MACS cell isolation system (Miltenyi Biotec, Bergisch Gladbach, Germany) as CD14-CD3-CD56+ cells [15]. Namely, the $\mathrm{CD}_{14}{ }^{+}$cells and $\mathrm{CD}^{+}$cells were depleted from PBMCs by incubation with anti-CD14 and anti-CD3 mAb-coupled magnetic beads, and then the $\mathrm{CD} 56^{+}$cells were positively selected by incubation with anti-CD56 mAbcoupled magnetic beads, according to the manufacturer's protocol. The sorted fraction contained $>99.6 \pm 0.2 \%$
CD56 ${ }^{+}$cells, and contamination with $\mathrm{CD}^{+}$cells was $<0.3$ $\pm 0.3 \%$. In some experiments, $T$ cells were also isolated as $\mathrm{CD}^{-} 6^{-} \mathrm{CD}^{+}{ }^{+}$cells using the MACS cell isolation system.

\section{Activated status of natural killer cells}

PBMCs were incubated with the following combination of fluorescently labeled mAbs: anti-CD56-fluorescein isothiocyanate, anti-CD69-phycoerythrin-cyanin 5.1, and anti-CD3-allophycocyanin (Beckman-Coulter, Fullerton, CA, USA). Fluorescent cell staining was detected by a FACSCalibur flow cytometer (Becton Dickinson, San Jose, CA, USA) using CellQuest ${ }^{\mathrm{TM}}$ software. Appropriate fluorescently labeled isotype-matched mAbs to irrelevant antigens were used in all analyses. The proportion of activated NK cells was assessed from the cells expressing CD69, an early activation marker of lymphocytes [18], within the CD56 ${ }^{+} \mathrm{CD} 3-\mathrm{NK}$ cell fraction.

\section{Cytotoxic activity}

The nonspecific cytotoxic activity of NK cells was quantified by a flow cytometry-based assay using NKTEST ${ }^{\circ}$ (Orpegen Pharma, Heidelberg, Germany). Briefly, K562 target cells pre-stained with a lipophilic green fluorescent membrane dye were mixed with freshly isolated effector PBMCs at an effector-to-target ratio of 25:1 and were incubated for 2 hours at $37^{\circ} \mathrm{C}$. Dead cells were detected by incubation with a DNA staining solution and subsequent analysis on a flow cytometer. The specific cytotoxicity (\%) was determined by subtracting the proportion of dead cells in the mock-treated sample from the proportion in the sample pre-treated with effector cells.

\section{Expression of genes associated with NK1/NK2 phenotype and cytotoxicity}

The total RNA was extracted from MACS-sorted NK cells using an RNeasy mini kit (Qiagen, Hilden, Germany), and was subjected to oligo (dT)-primed reverse transcription to generate first-strand cDNA. The cDNA equivalent to $5 \mathrm{ng}$ total RNA was subjected to semiquantitative PCR to detect IL-12R $\beta 2$, IFN $\gamma$, IL-5, IL-10, IL-13, perforin, granzyme $\mathrm{B}$, and glyceraldehyde-3-phosphate dehydrogenase (GAPDH), using specific primer sets as described elsewhere [15]. The PCR products were fractionated on agarose gels and visualized by ethidium bromide staining. The intensity of individual bands was semiquantitatively analyzed using the Image/ $\mathrm{J}^{\circ}$ software [19]. The relative expression level of individual genes was normalized to the expression of GAPDH.

The mRNA expression of selected genes was further evaluated using a quantitative Taqman real-time PCR system (Applied Biosystems, Foster City, CA, USA). All primers and probes were purchased from Applied Biosystems. The gene expression was standardized based on serial amounts of cDNA prepared from a healthy donor's 
PBMCs that were stimulated with phorbol 12-myristate13-acetate and ionomycin [15]. The relative expression levels of individual genes were normalized to the expression level of GAPDH.

\section{Phosphorylation status of signal transducer and activator of transduction 4}

The phosphorylated signal transducer and activator of transduction 4 (Stat4) and total Stat4 in IL-12-stimulated NK cells was detected by immunoblots as previously described [15]. The antibodies used were rabbit antiphosphorylated-Stat4 polyclonal antibodies (Zymed Laboratories, South San Francisco, CA, USA) and rabbit antiStat4 polyclonal antibodies (Santa Cruz Biotechnology, Santa Cruz, CA, USA). The intensity of individual bands with the expected molecular sizes was semiquantitatively analyzed using the image $/ \mathrm{J}^{\circ}$ software. The phosphorylation status of Stat 4 was expressed as the ratio of the intensity of phosphorylated Stat 4 to that of total Stat4.

\section{IFNy expression in CD4+ T cells co-cultured with natural killer cells}

The capacity of NK cells to modulate the expression of IFN $\gamma$ by $\mathrm{T}$ cells was evaluated using a cell-contact-free co-culture system. Briefly, MACS-sorted T cells $\left(2 \times 10^{6}\right)$ obtained from aBD patients were cultured in RPMI1640 supplemented with $7.5 \%$ low IgG fetal bovine serum (HyClone, South Logan, UT, USA) with or without sorted NK cells $\left(5 \times 10^{5}\right)$ prepared from iBD patients or healthy controls, applied to the upper chamber of an insert separated by a $0.4 \mu \mathrm{m}$ pore-size membrane (BD Biosciences, San Jose, CA, USA) on 12-well plastic plates, for 12 hours at $37^{\circ} \mathrm{C}$. Leukocyte Activation Cocktail $(5 \mu \mathrm{l} /$ well; BD Biosciences) was added at the initiation of the culture. The $T$ cells were then fixed and permeabilized using an Intracellular Cytokine Staining Kit Human (BD Biosciences), and were subsequently stained with anti-IFN $\gamma$ phycoerythrin (BD Biosciences) and anti-CD4-phycoerythrin-cyanin 5.1 (Beckman-Coulter), according to the manufacturer's protocols. The appropriate fluorescently labeled control antibodies were used to define the background Immunofluorescence of the cells. Finally, the cells were subjected to flow cytometry, and the IFN $\gamma$ expression level on the gated $\mathrm{CD} 44^{+} \mathrm{T}$ cells was calculated as a mean fluorescence intensity using CellQuest ${ }^{\mathrm{tm}}$ software. The relative IFNy expression was calculated as the ratio of IFN $\gamma$ expression by $\mathrm{CD} 4{ }^{+} \mathrm{T}$ cells cultured with $\mathrm{NK}$ cells to the expression by $\mathrm{CD} 44^{+} \mathrm{T}$ cells cultured alone.

\section{Statistical analysis}

All results are expressed as the mean \pm standard deviation. Statistical comparisons between two groups were performed using the Mann-Whitney $U$ test. Serial mea- surements were statistically evaluated by the Wilcoxon $t$ test.

\section{Results}

Clinical features of Behçet's disease patients

Of 47 patients with BD, $100 \%, 61 \%, 96 \%$, and $28 \%$ had had oral ulcer, uveitis, skin lesion, and genital ulcer, respectively, during the course of the disease. Only a small proportion of the patients had history of intestinal (6\%), vascular (11\%), and neurological (6\%) involvement. HLAB51 was detected in 28 patients $(60 \%)$. Treatment at the time of blood sampling included colchicine $(n=13)$, lowdose prednisolone $(n=3)$, cyclosporine $(n=2)$, etanercept $(\mathrm{n}=1)$, colchicine and low-dose prednisolone $(\mathrm{n}=$ 4), low-dose prednisolone and methotrexate $(n=1)$, colchicine, low-dose prednisolone and cyclosporine $(\mathrm{n}=$ 1), colchicine, low-dose prednisolone and azathioprine (n $=1$ ), and colchicine, low-dose prednisolone and infliximab $(n=1)$. Twenty patients $(42 \%)$ received no treatment.

Ten patients $(21 \%)$ were classified as having aBD at the time of blood sampling, based on a major uveitis attack (n $=7$ ), intestinal flare with a minor uveitis attack $(n=2)$, or exacerbation of mucocutaneous symptoms with high fever $(n=1)$. None of the aBD patients had concomitant flare of vascular or neurological involvement. There was no difference in the frequency of HLA-B51 or treatment regimens between $\mathrm{aBD}$ and $\mathrm{iBD}$. Seven patients with uveitis attack were treated with infliximab $(n=4)$, cyclosporine $(n=1)$, or an increased dosage of prednisolone in combination with cyclosporine $(n=2)$, resulting in resolution of symptoms within 3 months. Two patients with intestinal flare were treated with infliximab, resulting in resolution of all intestinal symptoms within 3 months. The mucocutaneous flare in the remaining patient was improved by high-dose prednisolone in combination with an increase in the dosage of cyclosporine.

\section{Activation status of natural killer cells}

We determined the activation status of the circulating NK cells in seven patients with aBD, 22 patients with iBD, and 19 healthy controls by examining the CD69 expression on the NK cells. As shown in Figure 1, the proportion of $\mathrm{CD}^{2} 9^{+}$-activated NK cells was significantly greater in the aBD patients than in the iBD patients or healthy controls $(P=0.01$ and $P=0.003$, respectively). There was a trend toward an increased proportion of activated NK cells in the iBD patients compared with in healthy controls, but the difference did not reach statistical significance $(P=$ 0.1 . These findings indicate that in vivo activation of circulating NK cells is observed in patients with aBD, but is not remarkable in those with iBD. 
A

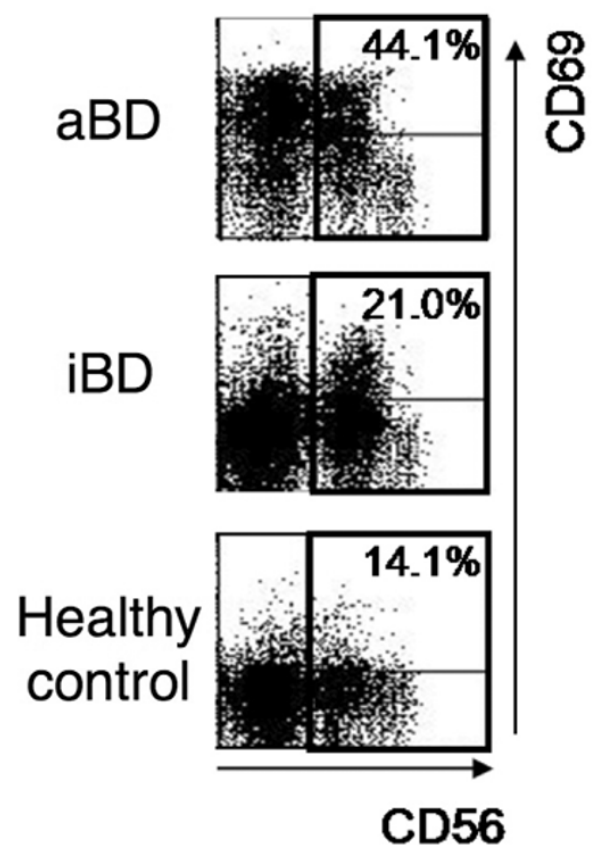

\section{B}

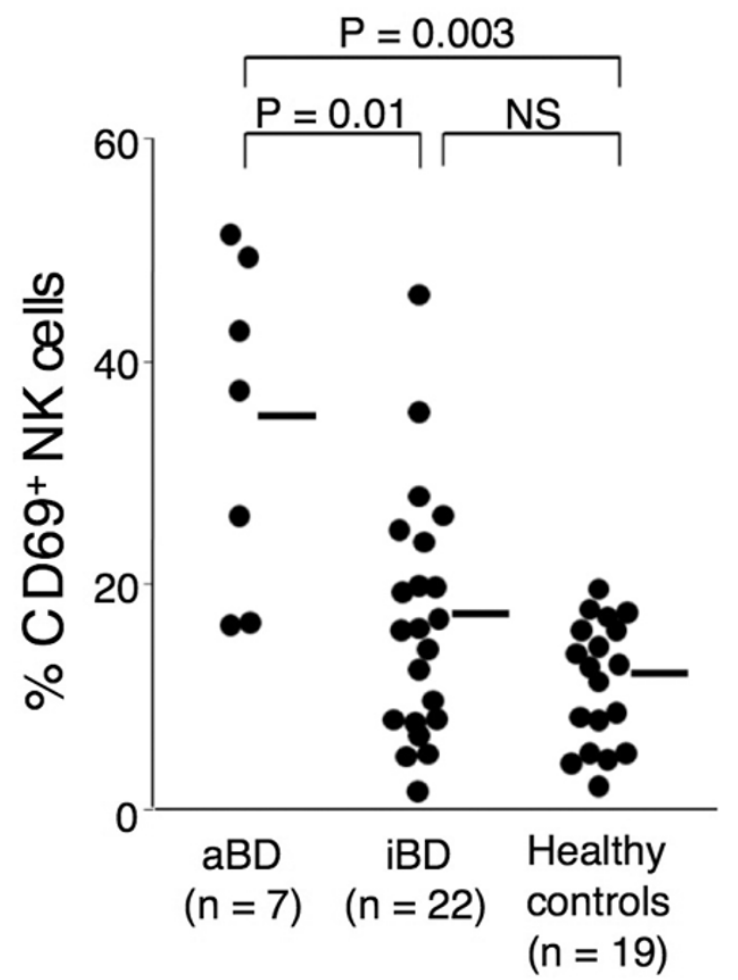

Figure 1 Activation status of natural killer cells in Behçet's disease. Proportion of activated natural killer (NK) cells in active Behçet's disease (aBD) patients, inactive Behçet's disease (iBD) patients, and healthy controls. (a) Representative dot-plot analysis for the expression of CD69 and CD56 in the gated CD3-lymphocytes from a patient with $\mathrm{BBD}$, a patient with $\mathrm{BDD}$, and a healthy control. The numbers indicate the proportion of CD69+-activated cells in total NK cells. (b) Proportion of CD69+-activated NK cells in seven aBD patients, $22 \mathrm{iBD}$ patients, and 19 healthy controls. Horizontal bars, mean values. NS, not significant.

\section{Cytotoxic activity of natural killer cells}

There was no difference in the nonspecific cytotoxic activity among the NK cells from three patients with aBD, 10 patients with iBD, and 13 healthy controls (14.9 \pm $10.3 \%, 14.3 \pm 5.4 \%$, and $14.4 \pm 7.4 \%$, respectively).

\section{Gene expression profiles of natural killer cells}

NK cells freshly isolated from aBD patients, iBD patients, and healthy controls were first subjected to semiquantitative PCR to measure the expression of genes associated with NK1/NK2 differentiation and cytotoxicity, including those encoding IL-12R $\beta 2$, IL-5, IL-10, IL-13, IFN $\gamma$, perforin, and granzyme B. Of these molecules, the mRNA levels of IL-12R 32 , perforin, and granzyme B were significantly lower, and that of IL-13 was significantly higher, in the iBD patients than in the aBD patients or healthy controls $(P<0.05$ for all comparisons) (data not shown). No IL-5 expression was detected in any of the samples from BD patients or healthy controls, and there was no statistically significant difference in the expression level of IL-10 or IFN $\gamma$.

To confirm the results obtained by semiquantitative PCR, the gene expression levels of IL-12R $\beta 2$, IL-13, perforin, and granzyme $\mathrm{B}$ were further evaluated by quantitative TaqMan real-time PCR (Figure 2). The IL-12R $\beta 2$ expression was significantly lower in the $\mathrm{BBD}$ patients than in the aBD patients or healthy controls $(P=0.006$ and $P=$ 0.0002 , respectively). The increased IL-13 expression was detected in a subset of patients with BD: most of them had iBD. The IL-13 expression level in iBD patients was significantly higher than the level in the healthy controls $(P=0.04)$, and tended to be higher than the level in aBD patients $(P=0.2)$. Interestingly, differences in IL-12R $\beta 2$ and IL-13 levels were not detectable between the aBD patients and healthy controls.

These findings indicated that the NK cells from iBD patients have a gene expression profile compatible with NK2; that is, upregulated IL-13 and downregulated IL- 

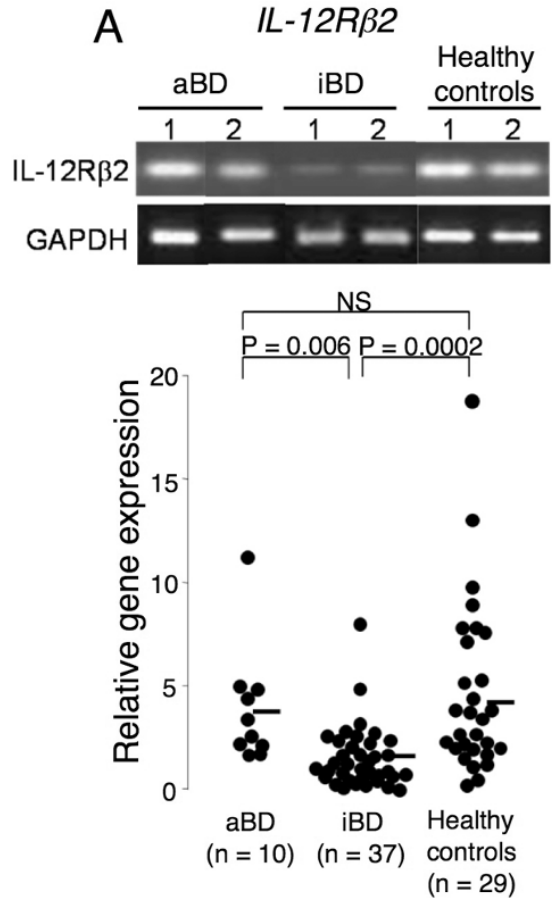

C
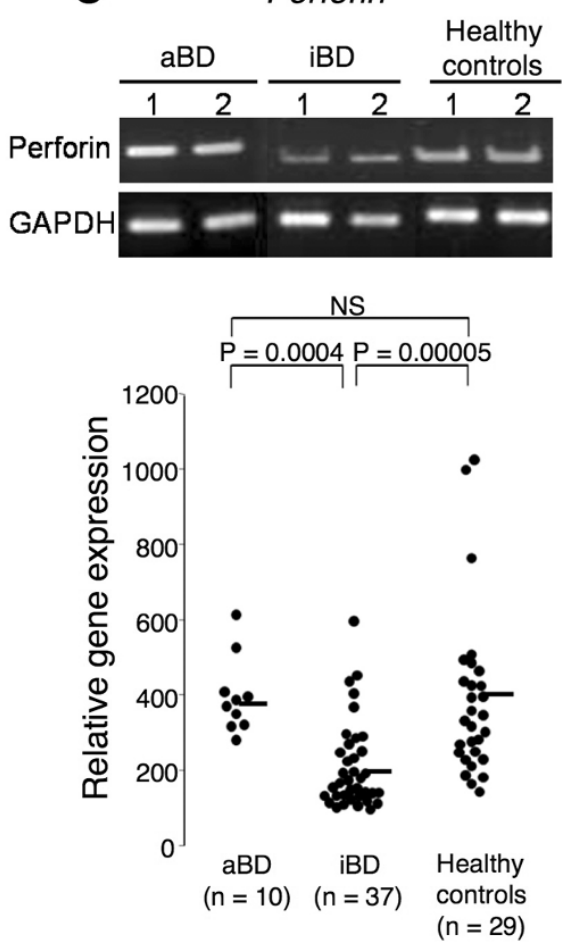
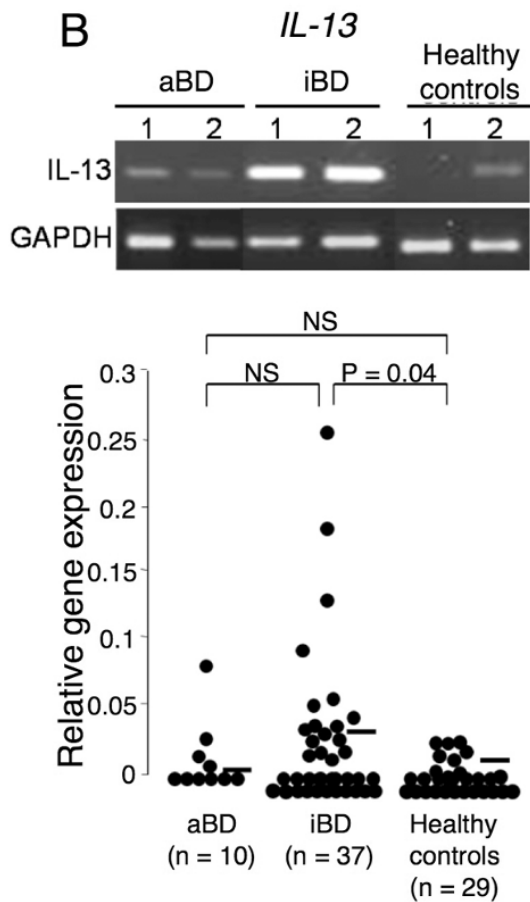

D Granzyme B
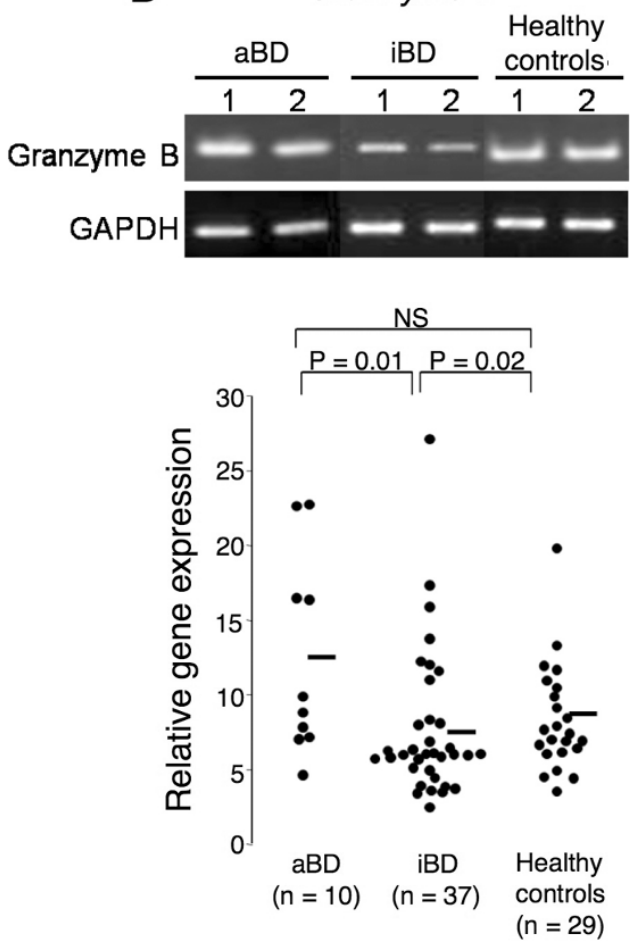

Figure 2 Gene expression levels of natural killer cells in Behçet's disease. Gene expression levels of (a) interleukin-12 receptor $\beta_{2}$ (IL-12Rß2), (b) IL-13, (c) perforin, and (d) granzyme B in natural killer (NK) cells from active Behçet's disease patients (aBD), inactive Behçet's disease (iBD) patients, and healthy controls. The IL-12Rß2, IL-13, perforin, and granzyme B expression levels in the NK cells were evaluated using semiquantitative PCR: two representative images each from aBD patients, $\mathrm{BD}$ patients, and healthy controls are shown in the upper portion of each panel. The relative gene expression levels were further determined by quantitative Taqman "real-time PCR in 10 aBD patients, 37 iBD patients, and 29 healthy controls: results are shown in the lower portion of each panel. Horizontal bars, mean values. GADPH, glyceraldehyde-3-phosphate dehydrogenase; NS, not significant. 
$12 R \beta 2$. On the other hand, the expression levels of perforin and granzyme $B$ were significantly lower in the $\mathrm{iBD}$ patients than in the aBD patients or healthy controls $(P$ $<0.02$ for all comparisons), while these expression levels were similar between these aBD patients and healthy controls.

\section{Serial gene expression analysis of natural killer cells}

For five aBD patients, additional blood samples were available when their BD symptoms were resolved after the introduction of infliximab $(n=2)$ or cyclosporine $(n=$ 1 ), or of an increased dosage of prednisolone in combination with cyclosporine $(n=2)$. The gene expression level of IL-12R $\beta 2$ was reduced in all five patients as the disease status became quiescent $(P=0.04)$ (Figure 3). IL-13 expression became detectable in three of the patients, and the change was borderline but did not reach a statistical significance $(P=0.05)$. These results strongly suggest that an NK2 shift was associated with disease remission. In addition, the expression level of perforin was reduced when the patients' disease status changed to remission $(P=0.02)$.

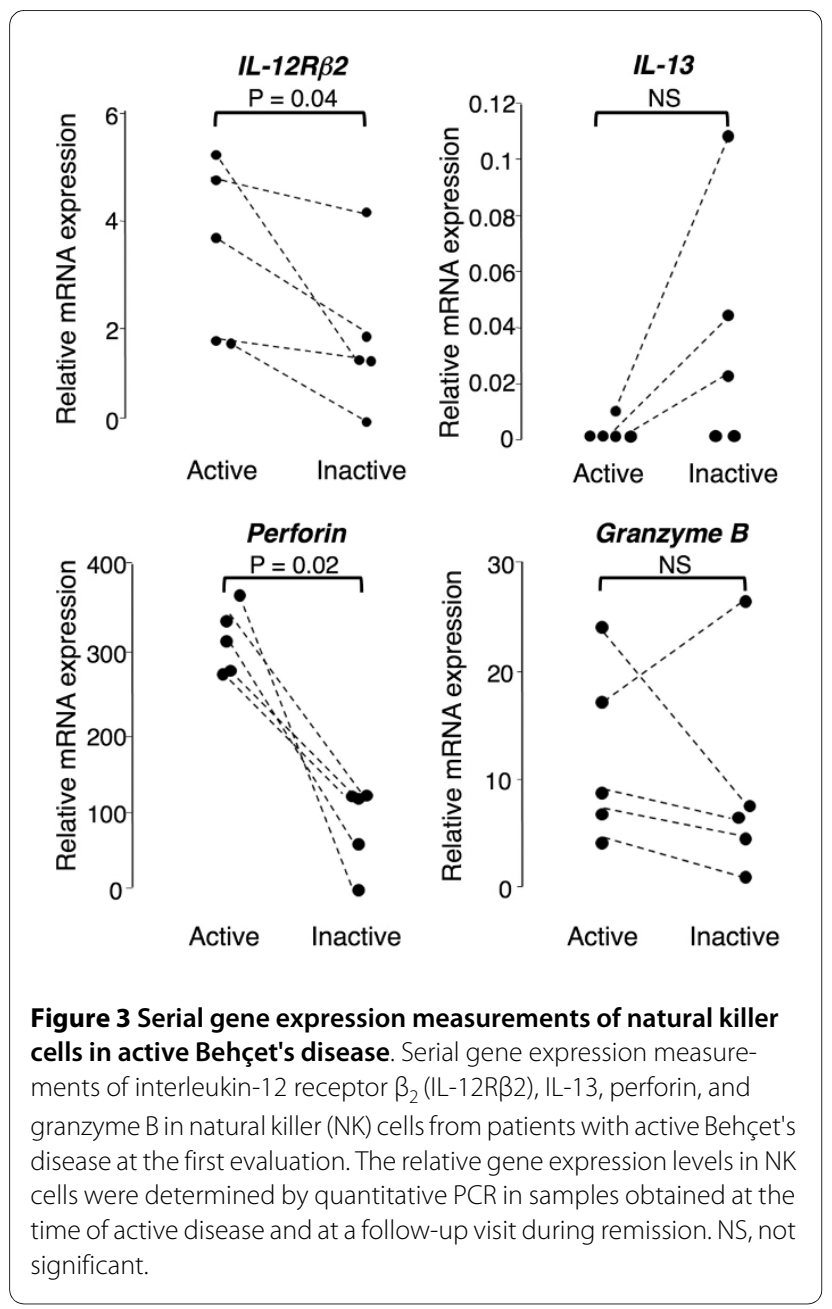

Impaired IL-12 signaling in natural killer cells from inactive Behçet's disease patients

The downregulated IL-12R $\beta_{2}$ gene expression observed in the NK cells from iBD patients could lead to impaired IL-12 signaling. To test this possibility, the phosphorylation status of Stat4, which is a downstream component of the IL-12 signaling pathway [20], was evaluated in the NK cells from six iBD patients and five healthy controls. As shown in Figure 4, the IL-12-induced Stat4 phosphorylation was significantly lower in the iBD patients than in the healthy controls $(P=0.02)$.

\section{Capacity of natural killer cells from inactive Behçet's disease patients to suppress IFN $\gamma$ expression by Th 1 cells} The NK2 bias observed in patients with iBD led us to speculate that NK cells play a role in controlling the pathogenic Th1 response in BD patients. To evaluate this hypothesis, the NK cells from iBD patients or healthy controls were co-cultured with Th1 cells derived from $\mathrm{aBD}$ patients in a cell-contact-free system. The intracellular IFN $\gamma$ expression in the gated $\mathrm{CD}^{+}{ }^{+} \mathrm{T}$ cells was then analyzed using flow cytometry (Figure 5). We found that the level of IFN $\gamma$ expressed by Th1 cells was reduced after

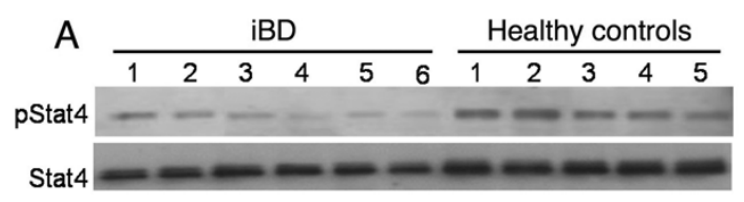

B

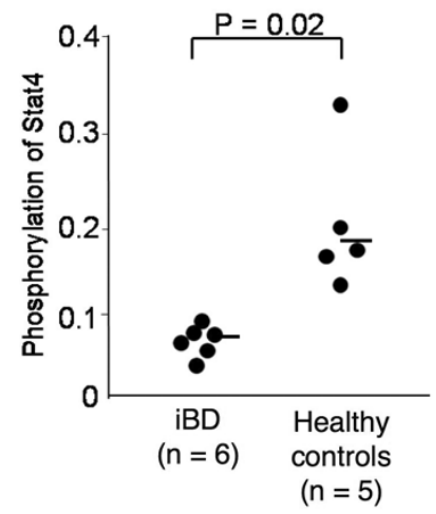

Figure 4 IL-12 signaling of natural killer cells in inactive Behçet's disease patients. IL-12-induced signal transducer and activator of transduction 4 (Stat4) phosphorylation in natural killer (NK) cells from inactive Behçet's disease (iBD) patients and healthy controls. (a) NK cells from six iBD patients and five healthy controls were stimulated with IL-12, and were subjected to immunoblotting for the detection of phosphorylated Stat4 (pStat4) and total Stat4. (b) Phosphorylation status of Stat4, which is expressed as the ratio of the intensity of pStat 4 to that of total Stat 4 , in NK cells from six iBD patients and five healthy controls. Horizontal bars, mean values. 


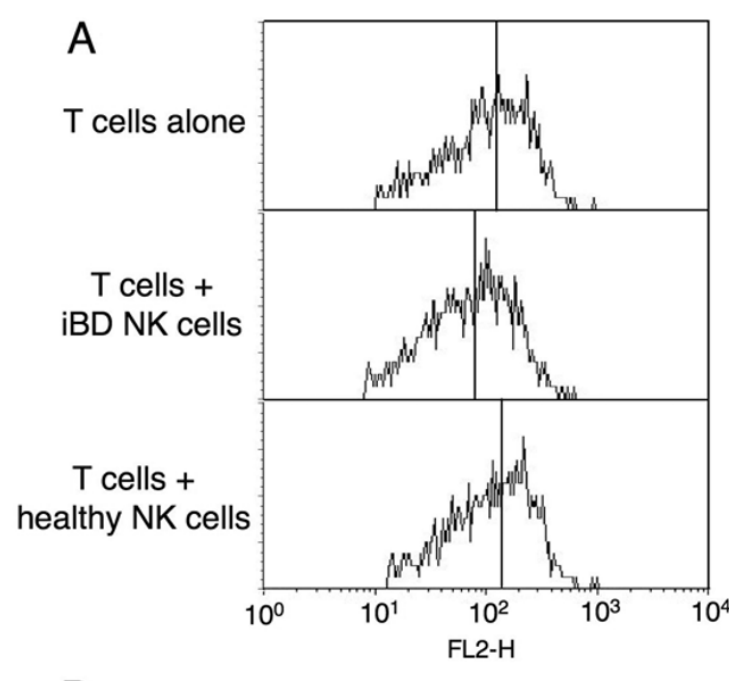

B

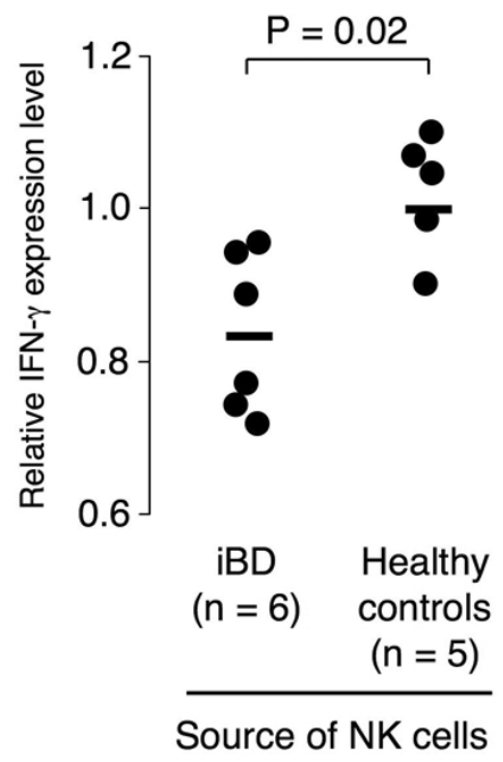

Figure 5 Natural killer cell suppression of IFNy expression by Thelper 1 cells in inactive Behçet's disease. Suppression of IFNY expression in T-helper 1 (Th1) cells by cell-contact-free co-culture with natural killer (NK) type 2 cells from inactive Behçet's disease (iBD) patients. T cells from active Behçet's disease patients were cultured alone or in combination with NK cells from healthy controls or iBD patients, and the IFNy expression level in the CD4+T cells was evaluated using flow cytometry. (a) Representative histogram plots showing expression of IFNy on gated CD4+ $T$ cells that were cultured alone, or with NK cells from an iBD patient or a healthy control. Vertical line in each histogram indicates the median. (b) IFNy expression levels in Th1 cells cultured with the NK cells from six iBD patients or from five healthy controls. Relative IFNY expression level calculated as the ratio of IFNY expression by $\mathrm{CD}_{4}{ }^{+} \mathrm{T}$ cells cultured with NK cells to the expression by $\mathrm{CD} 4+\mathrm{T}$ cells cultured alone. Horizontal bars, mean values. their co-culture with the NK cells derived from iBD patients. In fact, the relative IFN $\gamma$ expression level was significantly lower in the Th1 cells co-cultured with iBD patients' NK cells compared with the level in those cocultured with healthy controls' NK cells $(P=0.02)$. These findings suggest that the NK2 cells from $\mathrm{BBD}$ patients can suppress the Th1 response in aBD patients without cognate cell-cell contact.

\section{Discussion}

The present study has shown that the NK cells were phenotypically altered in BD patients, especially those in inactive disease status. Features of the circulating NK cells in iBD patients included: downregulated gene expression of IL-12R $\beta_{2}$; upregulated gene expression of IL-13 in a subset of the patients; downregulated perforin and granzyme B gene levels; and impaired IL-12-induced Stat4 phosphorylation. Upregulated IL-13 and downregulated IL-12R $\beta 2$ observed in NK cells from iBD patients were compatible with the NK2 phenotype. A serial NK phenotype analysis in $\mathrm{aBD}$ patients supported the association between NK2 bias and inactive disease status. Furthermore, NK2 cells obtained from iBD patients directly suppressed the IFN $\gamma$ expression of Th1 cells derived from aBD patients in vitro. These findings together suggest that the NK1/NK2 balance modulates disease flare/ remission in $\mathrm{BD}$ patients by controlling the pathogenic Th1 response. This situation is analogous to multiple sclerosis, another Th1-mediated disease, in which NK2 bias is associated with disease remission [13]. A major limitation of this study is the small number of patients analyzed, especially those with aBD. During 2 years of the study period, only 10 patients with aBD were enrolled in two major university hospitals in the Tokyo metropolitan area. In addition, there was a limited chance of obtaining peripheral blood samples from patients with $a B D$, because such patients required immediate introduction of treatment. Further multicenter studies involving a large number of patients with $\mathrm{aBD}$ are necessary to confirm our findings. Another limitation is the difficulty in classifying BD patients into those with active disease and those with inactive disease. We used a strict definition to select patients with aBD: flare of characteristic BD symptoms that required introduction of the intensive treatment, such as high-dose corticosteroids, cyclosporine, and infliximab. Patients with mild mucocutaneous manifestations or minor uveitis attack, which did not require intensive therapy, were therefore classified as having iBD. This clinical heterogeneity in the iBD subset may result in variability in the gene and protein expression profiles. Additional analysis according to individual clinical manifestations and/or treatment regimens would clarify these issues, but again the number of patients enrolled was too small to conduct subanalysis. Finally, we should recognize 
that a series of experiments involved only a subset of the patients and controls, which potentially bias the results. Our results suggest that the NK2 cells in iBD patients can suppress the Th1 response through at least two distinct mechanisms. First, the NK2 cells in iBD patients were intrinsically hyporesponsive to IL-12 due to their downregulated expression of IL-12R $\beta_{2}$ and impaired IL-12 signaling, resulting in deficient IFNY production even in the Th1 environment. Second, the NK2 cells from iBD patients actively suppressed IFN $\gamma$ expression in aBDderived Th1 cells. A similar inhibitory effect of human NK2 cells on the production of IFNY by T cells was also reported for healthy individuals' NK cells that were induced to express the NK2 phenotype [13], and for NK2 cells obtained from multiple sclerosis patients in remission [21]. Taken together, the NK cells and T cells - two major IFN $\gamma$ producers - were deficient in IFN $\gamma$ production in the NK2-biased immune environment observed in iBD patients.

How the NK2 cells from iBD patients suppress the IFN $\gamma$ expression in Th1 cells, however, remains unclear. One potential soluble mediator in our cell-contact-free culture system is IL-13, a typical T-helper 2 cytokine that inhibits Th1 responses in vitro and in vivo [22,23], although upregulated IL-13 expression was detected only in onethird of the iBD patients. In addition, this IL-13-mediated inhibitory effect is reported to occur predominantly through the modulation of antigen-presenting cells rather than as a direct effect on T cells [22]. Additional soluble factors secreted from NK2 cells are likely to be involved in this regulation, but the NK cells from iBD patients did not express IL-5, which plays a primary role in Th1 inhibition in multiple sclerosis patients in remission [13]. Furthermore, it has been reported that NK cells modulate Th1 responses also by interacting directly with $\mathrm{T}$ cells, $\mathrm{B}$ cells, and dendritic cells though cognate cell-cell contact $[24,25]$.

Perforin and granzyme B, major cytoplasmic granule toxins, were downregulated in the NK cells from patients with iBD. Interestingly, this gene expression profile is analogous to that of the NK cells in patients with active pemphigus vulgaris, who also show NK2 bias [15]. This phenomenon could be explained by the reduced IL-12R $\beta_{2}$ expression and impaired IL-12 signaling, but the cytotoxic activity was the same among the NK cells of iBD patients, $\mathrm{aBD}$ patients, and healthy controls. The reason for this inconsistency is unknown, but the cytotoxic activity of NK cells might be regulated by more complicated mechanisms, involving a balance between activating and inhibitory NK receptors, as well as the expression of the ligands for death receptors on target cells [26]. In aBD patients, the proportion of activated NK cells in the circulation was markedly increased. This is reasonable because IL-12 can activate NK cells in the Th1 envi- ronment, even though the nonspecific cytotoxic activity and gene expression profiles were similar between the NK cells from aBD patients and healthy controls. These activated NK cells would migrate to sites of inflammation and contribute to the ongoing tissue damage in aBD patients, but this appears to be just a bystander effect of the Th1 environment of aBD.

\section{Conclusions}

The present study is the first demonstrating a novel regulatory role for NK cells in the pathogenic process of BD. Our results have suggested that NK cells are actively involved in the induction and maintenance of disease remission in BD patients, through NK2 polarization. Future studies aimed at elucidating the mechanisms that control the NK1/NK2 paradigm in BD patients may be useful for developing new NK cell-targeted therapeutic strategies for BD.

\section{Abbreviations \\ aBD: active Behçet's disease; BD: Behçet's disease; GAPDH: glyceraldehyde-3- phosphate dehydrogenase; iBD: inactive Behçet's disease; IFN: interferon; IL: interleukin; IL-12R $\beta_{2}$ : interleukin-12 receptor $\beta_{2} ; \mathrm{mAb}$ : monoclonal antibody; NK: natural killer; NK1: natural killer type 1; NK2: natural killer type 2; PBMC: peripheral blood mononuclear cell; PCR: polymerase-chain reaction; Stat4: sig- nal transducer and activator of transduction 4; Th1: T-helper 1. \\ Competing interests \\ The authors declare that they have no competing interests. \\ Authors' contributions \\ YY performed the acquisition of data, and analysis and interpretation of the data, and wrote the manuscript. HT made a substantial contribution to the acquisition of data. TS and $\mathrm{YO}$ performed the acquisition of data. NM, KT, and ZI provided peripheral blood samples and clinical information, and performed analysis of the data. MK designed the experiments, performed data analysis and interpretation, and wrote the manuscript. All authors read and approved the final manuscript.}

\section{Acknowledgements}

The authors thank Aya Komori for their excellent technical assistance. The present work was supported by a research grant for intractable diseases from the Japanese Ministry of Health, Labour and Welfare (to MK).

\section{Author Details}

'Division of Rheumatology, Department of Internal Medicine, Keio University School of Medicine, 35 Shinanomachi, Shinjuku-ku, Tokyo 160-8582, Japan, 2Department of Environmental Immuno-Dermatology, Yokohama City University Graduate School of Medicine, 3-9 Fukuura, Kanazawa-ku, Yokohama 236-0004, Japan, ${ }^{3}$ Department of Dermatology, Keio University School of Medicine, 35 Shinanomachi, Shinjuku-ku, Tokyo 160-8582, Japan and

${ }^{4}$ Department of Ophthalmology, Yokohama City University Graduate School of Medicine, 3-9 Fukuura, Kanazawa-ku, Yokohama 236-0004, Japan

Received: 21 July 2009 Revised: 22 December 2009 Accepted: 11 May 2010 Published: 11 May 2010

\section{References}

1. Sakane T, Takeno M, Suzuki N, Inaba G: Behçet's disease. N Eng/ J Med 1999, 341:1284-1291.

2. Ben Ahmed M, Houman H, Miled M, Dellagi K, Louzir : Involvement of chemokines and Th 1 cytokines in the pathogenesis of mucocutaneous lesions of Behçet's disease. Arthritis Rheum 2004, 50:2291-2295. 
3. Frassanito MA, Dammacco R, Cafforio P, Dammacco F: Th1 polarization of the immune response in Behçet's disease: a putative pathogenetic role of interleukin-12. Arthritis Rheum 1999, 42:1967-1974.

4. Imamura Y, Kurokawa MS, Yoshikawa H, Nara K, Takada E, Masuda C, Tsukikawa S, Ozaki S, Matsuda T, Suzuki N: Involvement of Th1 cells and heat shock protein 60 in the pathogenesis of intestinal Behcet's disease. Clin Exp Immunol 2005, 139:371-378.

5. Yasuoka H, Okazaki Y, Kawakami Y, Hirakata M, Inoko H, Ikeda Y, Kuwana M: Autoreactive $\mathrm{CD}^{+}$cytotoxic T lymphocytes to major histocompatibility complex class I chain-related gene $A$ in patients with Behçet's disease. Arthritis Rheum 2004, 50:3658-3662.

6. Yasuoka H, Yamaguchi Y, Mizuki N, Nishida T, Kawakami Y, Kuwana M: Preferential activation of circulating $\mathrm{CD}^{+}$and $\gamma \delta \mathrm{T}$ cells in patients with active Behçet's disease and HLA-B51. Clin Exp Rheumatol 2008, 26:S59-S63.

7. Suzuki Y, Hoshi K, Matsuda T, Mizushima Y: Increased peripheral blood $\gamma \delta^{+} T$ cells and natural killer cells in Behçet's disease. J Rheumatol 1992, 19:588-592.

8. Kaneko F, Takahashi Y, Muramatsu R, Adachi K, Miura Y, Nakane A, Minagawa T: Natural killer cell numbers and function in peripheral lymphoid cells in Behcet's disease. Br J Dermatol 1985, 113:313-318.

9. Hamzaoui K, Ayed K, Hamza M, Touraine JL: Natural killer cells in Behcet's disease. Clin Exp Immunol 1988, 71:126-131.

10. Hamerman JA, Ogasawara K, Lanier LL: NK cells in innate immunity. Curr Opin Immunol 2005, 17:29-35.

11. Zhang C, Zhang J, Tian Z: The regulatory effect of natural killer cells: do 'NK-reg cells' exist? Cell Mol Immunol 2006, 3:241-254.

12. Peritt D, Robertson S, Gri G, Showe L, Aste-Amezaga M, Trinchieri G: Differentiation of human NK cells into NK1 and NK2 subsets. J Immunol 1998, 161:5821-5824.

13. Takahashi K, Miyake S, Kondo T, Terao K, Hatakenaka M, Hashimoto S, Yamamura T: Natural killer type 2 bias in remission of multiple sclerosis. $J$ Clin Invest 2001, 107:R23-R29.

14. Wei H, Zhang J, Xiao W, Feng J, Sun R, Tian Z: Involvement of human natural killer cells in asthma pathogenesis: natural killer 2 cells in type 2 cytokine predominance. J Allergy Clin Immunol 2005, 115:841-847.

15. Takahashi H, Amagai M, Tanikawa A, Suzuki S, Ikeda Y, Nishikawa T, Kawakami Y, Kuwana M: T helper type 2-biased natural killer cell phenotype in patients with pemphigus vulgaris. J Invest Dermatol 2007, 127:324-330.

16. International Study Group for Behçet's Disease: Criteria for diagnosis of Behçet's disease. Lancet 1990, 335:1078-1080.

17. Mizuki N, Ohno S, Ando H, Chen L, Palimeris GD, Stavropoulos-Ghiokas E, Ishihara M, Goto K, Nakamura S, Shindo Y, Isobe K, Ito N, Inoko H: A strong association of HLA-B*5101 with Behçet's disease in Greek patients. Tissue Antigens 1997, 50:57-60.

18. López-Cebrera M, Santis AG, Fernández-Ruiz E, Blacher R, Esch F, SánchezMateos P, Sánchez-Madrid F: Molecular cloning, expression, and chromosomal localization of the human earliest lymphocyte activation antigen AIM/CD69, a new member of the C-type animal lectin superfamily of signal-transmitting receptors. J Exp Med 1993, 178:537-547.

19. Image/J"s software [http://rsb.info.nih.gov/ii/index.html]

20. Chua AO, Wilkinson VL, Presky DH, Gubler U: Cloning and characterization of a mouse IL-12 receptor-beta component. Immunol 1995, 155:4286-4294.

21. Takahashi $\mathrm{K}$, Aranami T, Endoh M, Miyake S, Yamamura T: The regulatory role of natural killer cells in multiple sclerosis. Brain 2004 127:1917-1927.

22. Young DA, Lowe LD, Booth SS, Whitters MJ, Nicholson L, Kuchroo VK Collins M: IL-4, IL-10, IL-13, and TGF- $\beta$ from an altered peptide ligandspecific Th2 cell clone down-regulate adoptive transfer of experimental autoimmune encephalomyelitis. J Immunol 2000, 164:3563-3572.

23. Loza MJ, Peters SP, Zangrilli JG, Perussia B: Distinction between IL-13+ and IFN- $\gamma^{+}$natural killer cells and regulation of their pool size by IL-4. Eur J Immuno/ 2002, 32:413-423.

24. Mailliard RB, Son YI, Redlinger R, Coates PT, Giermasz A, Morel PA, Storkus WJ, Kalinski P: Dendritic cells mediate NK cell help for Th1 and CTL responses: two-signal requirement for the induction of NK cell helper function. J Immuno/ 2003, 171:2366-2373.
25. Zingoni A, Sornasse T, Cocks BG, Tanaka Y, Santani A, Lanier LL: NK cell regulation of T cell-mediated responses. Mol Immunol 2005, 42:451-454

26. Smyth MJ, Cretney E, Kelly JM, Westwood JA, Street SE, Yagita H, Takeda K, van Dommelen SL, Degli-Esposti MA, Hayakawa Y: Activation of NK cell cytotoxicity. Mol Immunol 2005, 42:501-510

doi: 10.1186/ar3005

Cite this article as: Yamaguchi et al., Natural killer cells control a T-helper 1 response in patients with Behçet's disease Arthritis Research \& Therapy 2010 12:R80

\section{Submit your next manuscript to BioMed Central} and take full advantage of:

- Convenient online submission

- Thorough peer review

- No space constraints or color figure charges

- Immediate publication on acceptance

- Inclusion in PubMed, CAS, Scopus and Google Scholar

- Research which is freely available for redistribution

Submit your manuscript at www.biomedcentral.com/submit
C Biomed Central 